\title{
HIGGS SEARCHES AT THE TEVATRON
}

\author{
KAZUHIRO YAMAMOTO for the CDF and D $\varnothing$ Collaborations \\ Department of Physics, Osaka City University, \\ Osaka 558-8585, Japan
}

\begin{abstract}
We present the latest results on searches for the standard and beyond-the-standard model Higgs bosons in proton-antiproton collisions at $\sqrt{s}=1.96 \mathrm{TeV}$ by the CDF and D $\varnothing$ experiments at the Fermilab Tevatron. No significant excess is observed above the expected background, and the cross section limits for the Higgs bosons are calculated. It is noticed that the standard model Higgs boson in the mass range $163-166 \mathrm{GeV} / c^{2}$ is excluded at the $95 \%$ C.L.
\end{abstract}

\section{Introduction}

The Higgs mechanism plays a role of a cornerstone in the modern particle physics. It was originally proposed to explain the non-zero masses of the weak bosons while keeping the theory gauge-invariant in the standard model (SM). As a result of the spontaneous electroweak symmetry braking, the weak bosons acquire the masses and simultaneously a new fundamental scalar particle, the Higgs boson $(H)$, appears. On the other hand in the minimal supersymmetric standard model (MSSM) which is one of the simplest extensions beyond the SM, two Higgs doublet fields result in five Higgs bosons denoted as $h, H, A, H^{ \pm}$. In spite of many experiments searching for these predicted particles, they have yet to be discovered. For the SM Higgs boson, the direct searches at the CERN LEP collider have set the lower limit on the Higgs boson mass $\left(M_{H}\right)$ to be $114.4 \mathrm{GeV} / \mathrm{c}^{2}$ at the 95\% C.L. [1]. Also combining this limit with precision electroweak measurements provides the constraint on $M_{H}$ to be less than $186 \mathrm{GeV} / c^{2}$ at the $95 \%$ C.L. [2]. The Tevatron experiments, $\mathrm{CDF}$ and $\mathrm{D} \emptyset$, are probing the Higgs boson in the most probable region, $100<M_{H}<200 \mathrm{GeV} / c^{2}$.

\section{Experimental Apparatus}

The Fermilab Tevatron collider is providing proton-antiproton collisions at $\sqrt{s}=1.96$ $\mathrm{TeV}$. The CDF and DØ detectors are general-purpose cylindrically symmetric detectors put into place at the Tevatron. Both detectors consist of a superconducting solenoid magnet, a precision tracking system, electromagnetic and hadronic calorimeters and muon spectrometers. The detailed description can be found elsewhere [3][4]. The accelerators and detectors keep running very well in stable condition. As of December 2009 , the typical peak luminosity is $3 \times 10^{32} \mathrm{~cm}^{-1} \mathrm{~s}^{-1}$ with the week integration of $50 \sim 60$ $\mathrm{pb}^{-1}$. The delivered luminosity to each detector is accumulated to be $7.4 \mathrm{fb}^{-1}$, among of which $6.1 \mathrm{fb}^{-1}$ is recorded on tape as the collision data. 


\section{Search for the Standard Model Higgs Boson}

There are three dominant processes for producing the SM Higgs boson at the Tevatron energy: gluon fusion $(g g \rightarrow H)$, associated production with a vector boson $\left(q \bar{q}^{(\text {() }} \rightarrow V H, V\right.$ $=W$ or $Z$ ), and vector boson fusion $\left(q \bar{q}^{(\prime)} \rightarrow q \bar{q}^{\left({ }^{\prime \prime}\right)} H\right)$. For the Higgs mass of $100 \sim 200$ $\mathrm{GeV} / \mathrm{c}^{2}$, the cross section of each process is predicted to be $0.2 \sim 1 \mathrm{pb}, 0.01 \sim 0.3 \mathrm{pb}$, and $0.02 \sim 0.1 \mathrm{pb}$ respectively [5]. As for the decay mode, $H \rightarrow b \bar{b}$ is dominant in the low mass case $\left(M_{H}<140 \mathrm{GeV} / c^{2}\right)$, while $H \rightarrow W W$ becomes dominant for the high mass Higgs $\left(M_{H}>140 \mathrm{GeV} / c^{2}\right)$. The Higgs searches are performed with the proper combinations of the above production and decay modes. Considering the huge backgrounds from QCD multijet production, it is appropriate to use $V H$ production with $H \rightarrow b \bar{b}$ for the low mass Higgs because a high- $P_{T}$ lepton and/or missing $E_{T}$ coming from the vector boson decay allows us to trigger the events efficiently. On the other hand for the high mass Higgs, we take advantage of the large cross section of $g g \rightarrow H$ followed by $H \rightarrow W W$. The analyses are optimized for every channel of the different topological signatures in terms of the decay mode and jet multiplicity. The event selections and analysis strategies of each Higgs search channel are described below. The data used here correspond to an integrated luminosity of $2.0 \sim 5.4 \mathrm{fb}^{-1}$. The obtained results on the cross section upper limit are summarized in Table I. The further details can be found in Refs. [6] - [15].

We search for $Z H \rightarrow v \bar{v} b \bar{b}$ by investigating the missing $E_{T}+b \bar{b}$ signature. Considering the charged leptons which escape from the detector, this signature is also sensitive to a portion of $W H \rightarrow \ell v b \bar{b}$ and $Z H \rightarrow \ell^{+} \ell^{-} b \bar{b}$. The event selections used in the CDF and D $\varnothing$ analyses are similar. They are performed by vetoing events with isolated charged leptons ( $e$ or $\mu$ ) and also by requiring large missing $E_{T}$ and 2 or 3 jets containing at least one $b$ tagged jet. For $b$-tagging, the secondary vertex tagger (SECVTX) and the jet probability tagger (JP) are utilized at CDF, while the neural network (NN) tagger is developed and used at $\mathrm{D} \emptyset$. The dominant backgrounds are $W / Z+$ jets, $t \bar{t}$, diboson $(W W, W Z, Z Z)$, and QCD multi-jets. The multivariate discriminant technique is applied in order to effectively separate signal from background; the CDF uses a NN while the D $\varnothing$ uses a boosted decision tree (BDT). The event separation is performed in two steps; the first discriminant is trained to remove the multi-jet background, and the second discriminant separates the expected signal from the remaining SM background.

The process $W H \rightarrow \ell v b \bar{b}$ is also a very sensitive channel in the low mass region as well as $Z H \rightarrow v \bar{v} b \bar{b}$. The events are selected by requiring a single isolated high- $p_{T}$ lepton, large missing $E_{T}$ and 2 or 3 energetic jets with at least one $b$-tagged jet. The dominant background in the selected events is $W b \bar{b}$ for the 2 jets sample and $t \bar{t}$ for the 3 jets sample. The other backgrounds include $W / Z+$ jets, single top, diboson and non- $W$ QCD jets. For the DØ analysis, a $\mathrm{NN}$ is employed to discriminate signal from background after the base selection. On the other hand for the CDF analysis, efforts to achieve better sensitivity are made by two analysis groups each of which employs a matrix element method (ME) and a NN respectively. The discriminant is optimized for each $b$-tag category.

Another important channel in the low mass region is $Z H \rightarrow \ell^{+} \ell^{-} b \bar{b}$. In the base selection, events containing $Z$ candidates reconstructed from $e^{+} e^{-}$and $\mu^{+} \mu^{-}$pairs are selected at first. Then, two or more energetic jets at least one of which is $b$-tagged are 
required. Backgrounds of this analysis are dominated by $Z+$ jets, $t \bar{t}, W Z, Z Z$, and QCD events where a hadron is misidentified as a lepton. In order to discriminate signal from background, a BDT is used in the DØ analysis, while a two dimensional $\mathrm{NN}$ is implemented in the CDF analysis, where $Z+$ jets and $t \bar{t}$ backgrounds are separated out from signal simultaneously.

The Higgs events of which final state includes a $\tau^{+} \tau^{-}$pair also become significant by combining all relevant channels. For the DØ analysis, the following five channels: $Z H\left(Z \rightarrow \tau^{+} \tau^{-}, H \rightarrow q \bar{q}\right), \quad H Z\left(H \rightarrow \tau^{+} \tau^{-}, Z \rightarrow q \bar{q}\right), H W\left(H \rightarrow \tau^{+} \tau^{-}, W \rightarrow q \bar{q}^{\prime}\right), q \bar{q}^{(\prime)} \rightarrow$ $H q \bar{q}^{\left({ }^{\prime}\right)}\left(H \rightarrow \tau^{+} \tau^{-}\right.$, vector boson fusion), and $g g \rightarrow H \rightarrow \tau^{+} \tau^{-}$(gluon fusion) are considered. Identification of $\tau^{+} \tau^{-}$decay is essential for this analysis, and events in which one $\tau$ decays leptonically and the other $\tau$ decays hadronically are selected in order to increase the signal to noise ratio. An isolated object consisting of one or three charged particles in a narrow cone is required as a signature of a hadronically decaying $\tau$, and a NN is used for further $\tau$ identification. After selecting the $\tau$ rich sample, an analysis using a BDT is performed to separate the expected signal from the remaining backgrounds which are mainly $t \bar{t}, W / Z+$ jets, and QCD multi-jets.

In the high mass region, $H \rightarrow W W^{*} \rightarrow \ell^{+} v \ell^{-} \bar{v}$ is the most sensitive channel at the Tevatron. The most dominant process for producing the Higgs boson in this channel is gluon fusion $(g g \rightarrow H)$. The other contribution comes from associated production with a vector boson $(W H, Z H)$ and vector boson fusion $\left(q \bar{q}^{(,)} H\right)$. The event selection is performed by requiring high- $P_{T}$ opposite-sign dilepton with large missing $E_{T}$. The main backgrounds are $t \bar{t}$, Drell-Yan, $W \gamma$, and $W+$ jets. Considering the scalar nature of the Higgs boson and the parity violation in $W$ decays, two charged leptons from the Higgs decay tend to go in the same direction, which is different from the SM backgrounds. The final discriminants are NN outputs which are obtained by feeding a number of kinematic variables as inputs. The opening angle between two leptons mentioned above is one of the effective input variables. The NN training is carefully performed for the separated subsamples; the CDF analysis separates the sample by the jet multiplicity ( 0 jet, 1 jet, 2 or more jets), while the $\mathrm{D} \varnothing$ analysis makes classification by dilepton flavor $(e e, \mu \mu, e \mu)$. Also the low dilepton mass region $\left(M_{\ell \ell}<16 \mathrm{GeV} / c^{2}\right)$ and the same-sign dilepton + jets sample $\left(W H \rightarrow W W W^{*}, Z H \rightarrow Z W W^{*}\right)$ are added to increase sensitivity. Due to the different background composition, the dedicated $\mathrm{NN}$ training is performed separately.

All the available results on the Higgs boson searches are combined into a single limit on the Higgs boson production cross section at the 95\% C.L. for each Higgs boson mass ranging from $100 \mathrm{GeV} / c^{2}$ to $200 \mathrm{GeV} / c^{2}$. The combination process is made in two steps; the results of various analysis channels are combined in each experiment of CDF and D $\varnothing$ separately at first [15][16], and then the both results are combined into the single Tevatron combination [17]. In calculating the combined result, systematic correlations among the analysis channels and also those between experiments are carefully taken into account. The obtained limit is presented as a ratio to the predicted cross section by the SM in Fig. 1. We obtain the observed (expected) values of 2.70 (1.78) at $M_{H}=115 \mathrm{GeV} / c^{2}$ and $0.94(0.89)$ at $M_{H}=165 \mathrm{GeV} / c^{2}$. We exclude the SM Higgs boson in the mass range $163 \mathrm{GeV} / c^{2}$ to $166 \mathrm{GeV} / c^{2}$, while the corresponding expected excluded region is $159 \mathrm{GeV} / c^{2}$ to $169 \mathrm{GeV} / c^{2}$. 
TABLE I: Observed (Expected) limits at the 95\% C.L. on the production cross section as a ratio to the SM Higgs cross section. The limits at $M_{H}=115 \mathrm{GeV} / c^{2}$ are presented for the first four processes, while $165 \mathrm{GeV} / c^{2}$ for $H \rightarrow W W$. Two analysis results are shown for the CDF $W H \rightarrow \ell v b \bar{b}$ channel; one is a result by using a neural network (NN) and the other is obtained with a matrix element method (ME).

\begin{tabular}{|c|c|c|c|c|c|}
\hline \multirow[b]{2}{*}{ Analysis channel } & \multirow[b]{2}{*}{$\begin{array}{c}M_{H} \\
\left(\mathrm{GeV} / c^{2}\right)\end{array}$} & \multicolumn{2}{|l|}{$\mathrm{CDF}$} & \multicolumn{2}{|l|}{$\mathrm{D} \varnothing$} \\
\hline & & $\begin{array}{c}\text { Observed (Expected) } \\
\text { limit }\left[\sigma / \sigma_{\mathrm{SM}}\right]\end{array}$ & $\begin{array}{c}\mathcal{L} \\
\left(\mathrm{fb}^{-1}\right)\end{array}$ & $\begin{array}{c}\text { Observed (Expected) } \\
\text { limit }\left[\sigma / \sigma_{\mathrm{SM}}\right]\end{array}$ & $\begin{array}{c}\mathcal{L} \\
\left(\mathrm{fb}^{-1}\right)\end{array}$ \\
\hline$V H \rightarrow \boldsymbol{E}_{T} b \bar{b}$ & 115 & $6.1(4.2)[6]$ & 3.6 & $3.7(4.6)[7]$ & 5.2 \\
\hline$W H \rightarrow \ell v b \bar{b}$ & 115 & $\begin{array}{l}5.3(4.0), \mathrm{NN}[8] \\
6.6(4.1), \mathrm{ME}[9]\end{array}$ & 4.3 & $6.9(5.1)[10]$ & 5.0 \\
\hline$Z H \rightarrow \ell^{+} \ell^{-} b \bar{b}$ & 115 & $5.9(6.8)[11]$ & 4.1 & $9.1(8.0)[12]$ & 4.2 \\
\hline$\tau^{+} \tau^{-} q \bar{q}$ final state & 115 & \multicolumn{2}{|l|}{ Update in progress } & $27.0(15.9)[13]$ & 4.9 \\
\hline$H \rightarrow W W$ & 165 & $1.23(1.21)[14]$ & 4.8 & $1.55(1.36)[15]$ & 5.4 \\
\hline
\end{tabular}

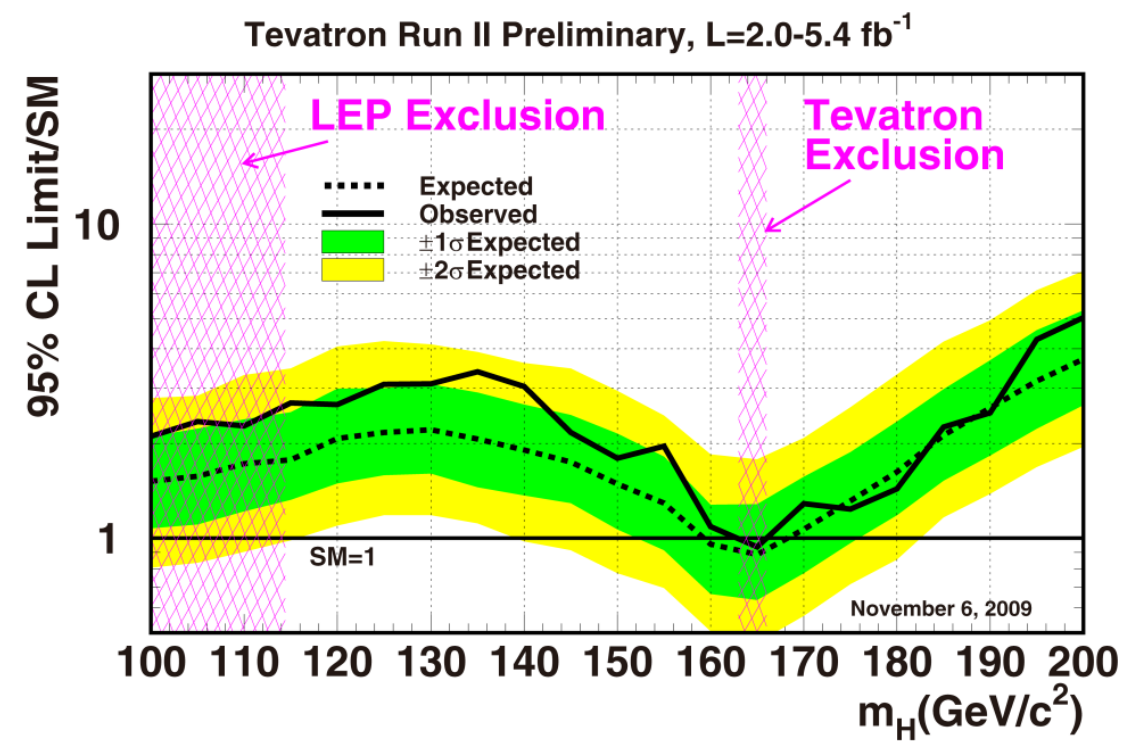

FIG. 1: Observed (solid line) and expected (dashed line) 95\% C.L. upper limits on the ratio to the SM Higgs cross section as functions of the Higgs boson mass for the combined CDF and $\mathrm{D} \emptyset$ analyses. The bands indicate the $68 \%$ and $95 \%$ probability regions where the limit is expected to fluctuate, in the absence of signal. 


\section{Search for the Higgs Bosons Beyond the SM}

The minimal supersymmetric standard model (MSSM) is one of the simplest extensions beyond the SM. The production cross section of the neutral Higgs bosons $(\phi=h, H, A)$ is enhanced by $\tan ^{2} \beta$. At the Tevatron energy, the production cross section is $10 \sim 50 \mathrm{pb}$ at $\tan \beta=40$ [5], which is one order of magnitude larger than the SM. The main decay modes of $\phi$ are $b \bar{b}(\sim 90 \%)$ and $\tau^{+} \tau^{-}(\sim 10 \%)$ in a wide mass range $\left(100 \sim 200 \mathrm{GeV} / c^{2}\right)$.

One of the most promising channels to search for the neutral MSSM Higgs bosons at the Tevatron is $\phi \rightarrow \tau^{+} \tau^{-}$in combination with the Higgs production through gluon fusion $(g g \rightarrow \phi)$ and $b \bar{b}$ fusion $(b \bar{b} \rightarrow \phi)$. Tau pairs are identified in decay modes of $e \mu$, $e+$ hadrons and $\mu+$ hadrons, where the hadronic $\tau$ decay is identified with the similar method as the SM Higgs search described in the previous section. The background is dominated by $Z \rightarrow \tau^{+} \tau^{-}$, diboson, $t \bar{t}$ and $W+$ jets. Also certain portion of the background arises from $Z \rightarrow e e / \mu \mu$ by misidentifying a lepton as a hadron. Figure 2 shows the combined result of the CDF and $\mathrm{D} \emptyset$ data corresponding to an integrated luminosity of $1.0 \sim 2.2 \mathrm{fb}^{-1}$. It presents the $95 \%$ C.L. upper limit on $\tan \beta$ as a function of the $A$ boson mass $\left(M_{A}\right)$ with an assumption of the typical MSSM scenario of the maximal stop mixing and $\mu=-200 \mathrm{GeV}[19]$.

Another explorable channel of $\phi$ is $g b \rightarrow b \phi \rightarrow b b \bar{b}$. Three $b$-tagged jets are required in the analysis, and a peak structure is searched for in the dijet mass spectrum above the large multi-jet background. Figure 3 shows the results released with $1.9 \mathrm{fb}^{-1}$ at CDF [20] and $2.6 \mathrm{fb}^{-1}$ at $\mathrm{D} \emptyset$ [21].

The charged MSSM Higgs $\left(H^{ \pm}\right)$bosons can be investigated in top quark decays; a top quark decays into not only $W b$ but also $H^{ \pm} b$ when $H^{ \pm}$is lighter than $t$. In this case, there would be some deviation from the SM prediction for the final states of $t \bar{t}$ decay. However, the results are consistent with the SM prediction at both CDF and DØ, and hence the upper limits are obtained as shown in Fig. 4 [22][23].

In some models beyond the SM, the neutral Higgs couplings to fermions are suppressed due to some specific mechanisms, thus the Higgs boson decay into vector bosons are significantly increased. This kind of "fermiophobic" Higgs boson mainly decays into $\gamma \gamma$ for the low mass region, while $W W$ and $Z Z$ become the main decay mode in the intermediate and high mass region. For simplicity, the benchmark scenario is

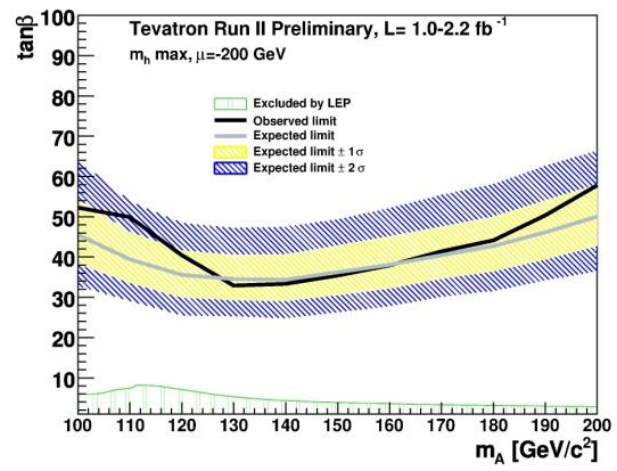

FIG. 2: The 95\% C.L. limits in the $\tan \beta-M_{A}$ plane for the typical MSSM scenario of the maximal stop mixing and $\mu=-200 \mathrm{GeV}$. The black line denotes the observed limit, the gray line denotes the expected limit, and the hatched regions indicate the $\pm 1 \sigma$ and $\pm 2 \sigma$ bands around the expectation. 
considered by assuming that the Higgs does not couple to any fermions but couples to electroweak bosons with the SM coupling strength. Figure 5 shows the results from CDF with $3.0 \mathrm{fb}^{-1}$ and DØ with $4.2 \mathrm{fb}^{-1}$ in $\gamma \gamma$ decay mode [24][25]. Both experiments exclude the fermiophobic Higgs boson with masses below $\sim 110 \mathrm{GeV} / c^{2}$.

(a)

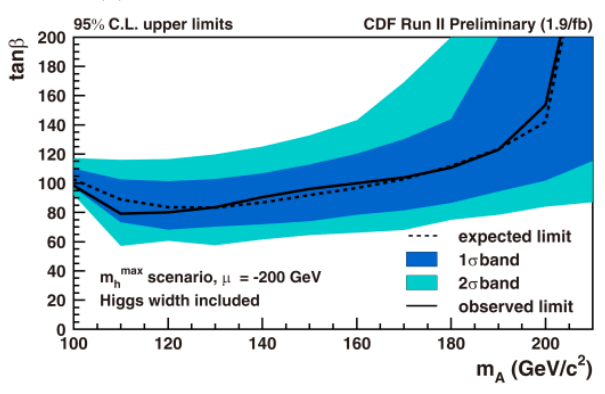

(b)

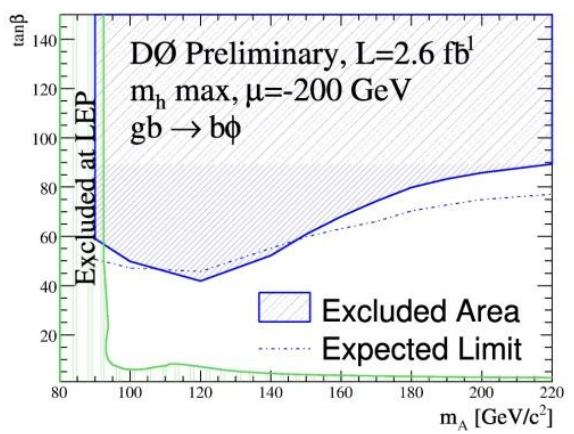

FIG. 3: The $95 \%$ C.L. upper limits in the $\tan \beta-M_{A}$ plane for the MSSM scenario of maximal stop mixing and $\mu=-200 \mathrm{GeV}$, which is obtained from the analyses of $g b \rightarrow b \phi \rightarrow b b \bar{b}$ channel. The left plot (a) is a result of the CDF analysis, and the right plot (b) is that of the $\mathrm{D} \emptyset$ analysis.

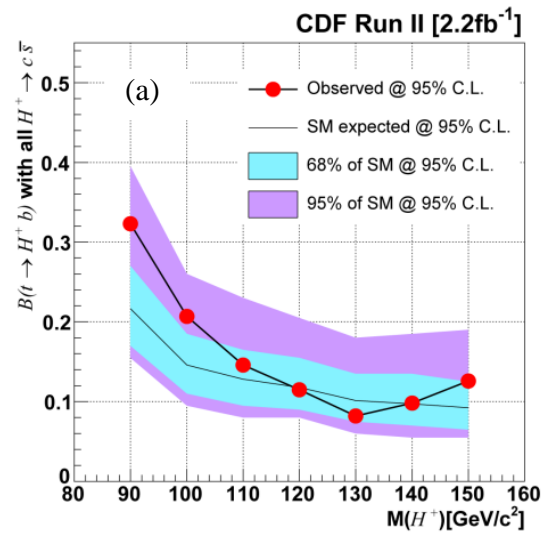

(b)

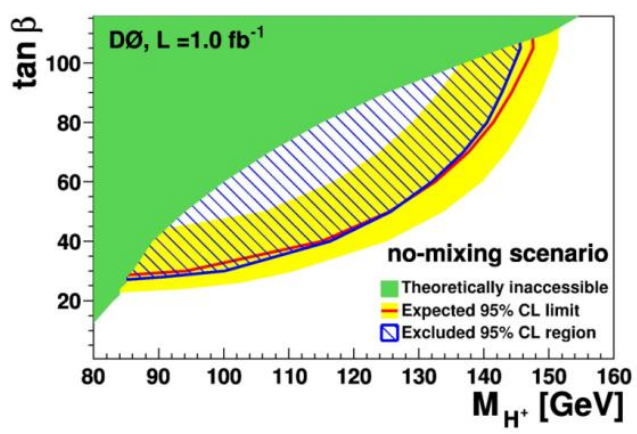

FIG. 4: The left plot (a) shows the CDF result of the 95\% C.L. upper limits on the branching fraction of $t \rightarrow H^{+} b$ as a function of $M_{H^{+}}$with an assumption that all $H^{+}$'s decay into $c \bar{s}$. The right plot (b) shows the D $\varnothing$ result of the excluded region in the $\tan \beta-M_{H^{+}}$plane at the $95 \%$ C.L. for the no-mixing scenario. 


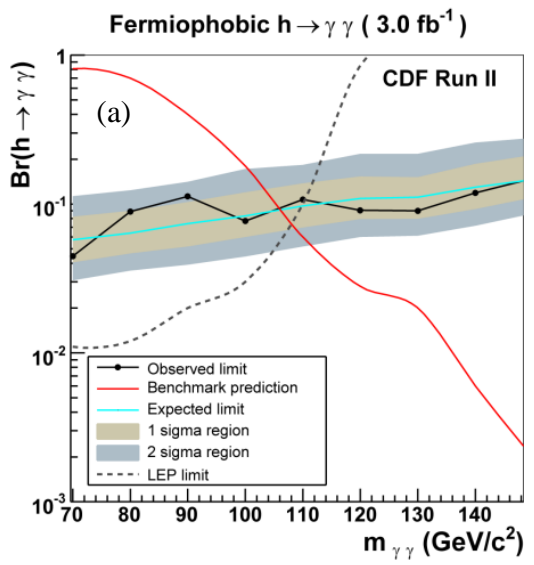

(b)

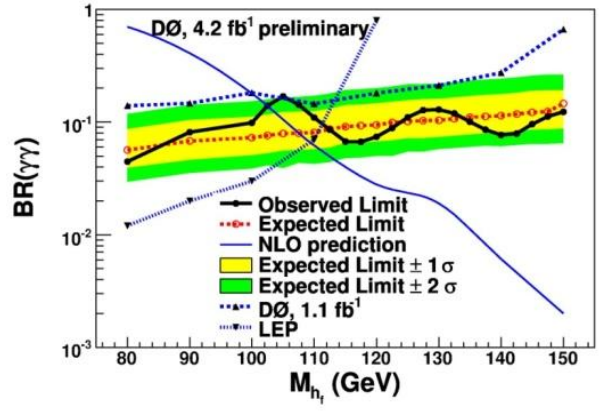

FIG. 5: The 95\% C.L. upper limits on the branching fraction for the fermiophobic Higgs boson decay to diphotons, as a function of the fermiophobic Higgs mass. The result on the left plot (a) is obtained at CDF and that on the right plot (b) is obtained at DØ.

\section{Conclusions and Future Prospects}

The Tevatron accelerators and the collider detectors, CDF and DØ, are performing very well. The integrated delivered luminosity as of December 2009 is $7.4 \mathrm{fb}^{-1}$, while $6.1 \mathrm{fb}^{-1}$ is acquired as the collision data on tape. The Higgs boson searches are in progress in the various production and decay channels. The preliminary results with up to $5.4 \mathrm{fb}^{-1}$ of data are presented in this report. For the SM Higgs boson, the combined results of CDF and DØ exclude the mass range from 163 to $166 \mathrm{GeV} / c^{2}$ at the $95 \%$ C.L., while the expected exclusion range is 159 to $168 \mathrm{GeV} / \mathrm{c}^{2}$. On the other hand for the Higgs bosons beyond the SM, no sign of discovery has been observed yet. However, the sensitivity is steadily increasing. By the end of FY2010, the luminosity is expected to be accumulated up to 9 $\mathrm{fb}^{-1}$, and the sensitivity for seeing the Higgs boson will significantly increase.

\section{Acknowledgements}

I would like to thank the members of the CDF and $D \emptyset$ collaborations for their work and effort in achieving the results presented in this report.

\section{References}

1. The ALEPH, DELPHI, L3 and OPAL Collaborations, and the LEP Working Group for Higgs Boson Searches, Phys. Lett. B 565, 61 (2003). 
2. The ALEPH, CDF, DØ, DELPHI, L3, OPAL, SLD Collaborations, the LEP Electroweak Working Group, the Tevatron Electroweak Working Group, and the SLD Electroweak and Heavy Flavor Groups, arXiv:0911.2604 [hep-ex] (2009).

3. T. Acosta et al. (CDF Collaboration), Phys. Rev. D 71, 032001 (2005).

4. V. M. Abazov et al. (DØ Collaboration), Nucl. Instr. Meth. A565, 463 (2006).

5. TeV4LHC Higgs Working Group, arXiv:hep-ph/0612172 (2007).

6. CDF Collaboration, CDF Note 9642 (2009).

7. DØ Collaboration, DØ Note 5872-CONF (2009).

8. CDF Collaboration, CDF Note 9997 (2009).

9. CDF Collaboration, CDF Note 9985 (2009).

10. DØ Collaboration, DØ Note 5972-CONF (2009).

11. CDF Collaboration, CDF Note 9889 (2009).

12. DØ Collaboration, D $\varnothing$ Note 5876-CONF (2009).

13. DØ Collaboration, D $\varnothing$ Note 5845-CONF (2009).

14. CDF Collaboration, CDF Note 9887 (2009).

15. DØ Collaboration, DØ Note 6006-CONF (2009).

16. CDF Collaboration, CDF Note 9999 (2009).

17. DØ Collaboration, DØ Note 6008-CONF (2009).

18. The TEVNPH Working Group, arXiv:0911.3930 [hep-ex] (2009).

19. The TEVNPH Working Group, FERMILAB-PUB-09-394-E, CDF Note 9888, DØ Note 5980-CONF (2009).

20. CDF Collaboration, CDF Note 9284 (2008).

21. DØ Collaboration, DØ Note 5726-CONF (2008).

22. T. Aaltonen et al. (CDF Collaboration), Phys. Rev. Lett. 103, 101803 (2009).

23. DØ Collaboration, DØ Note 5715-CONF (2008).

24. T. Aaltonen et al. (CDF Collaboration), Phys. Rev. Lett. 103, 061803 (2009).

25. DØ Collaboration, DØ Note 5880-CONF (2009). 